\section{Maxillofacial Trauma: Peri Operative Challenges in the Management of Facial Bone Fractures}

\section{Abstract}

Facial trauma and fractures of the facial bones may have associated brain and cervical spine injuries. Assault, gunshot wounds and motor vehicle accidents (MVA) are major contributory causes. Trauma units must be competent in airway management. Death may result from trauma itself, haemorrhage, aspiration or hypoxia. Initial screening of neurological injury must be followed by neural imaging studies and neurological examination.

Keywords: Facial bone fracture; Airway management; Cognitive assessment; High resolution brain scan; Definitive surgery delayed; Post-operative ventilation

Received: October 30, 2019; Accepted: October 30, 2019; Published: November 10, 2019

\section{Introduction}

Maxillofacial and neck trauma frequently present as emergencies. These injuries are daunting surgically and present unique challengers for anaesthesiologists. Airway management skills in head and neck trauma are essential as the anatomy may be distorted and compromised by bleeding and aspiration of blood and vomitus into the lungs [1].

The causes of facial injury in Southern Africa are:

\section{Interpersonal violence}

More prevalent in the young age group (20-39). Assault is the major cause of head trauma and maxillofacial trauma (60\%) with males being the predominant victims [2].

Some cities in South Africa (SA) being amongst the most violent in the world due to gang warfare, car high-jacking and home invasions. Alcohol consumption, femicide, domestic and genderbased violence is common with one in four women being a survivor [3].

\section{Traumatic injuries from motor vehicle accidents (MVA)}

These accounts for (17.5\%) of maxillofacial trauma [2]. SA has a of the poor road safety record. WHO 2018 report that the number annual road traffic deaths have reached 1.35 million with 3,287 deaths a day [4]. The Automobile Association of SA (AA) report 134,000 deaths over 10 years. The fatality rate globally is approximately $18 / 100000$ people and South African statistics $32 / 100000$ [5].

\section{Bulbulia BA*, Vally IM and Vally U \\ Ahmed Kathrada Private Hospital, Lenasia, South Africa}

\section{*Corresponding author: Bulbulia BA}

Đbash786@absamail.co.za

Ahmed Kathrada Private Hospital, Lenasia, South Africa.

Tel: 27834173527

Citation: Bulbulia BA, Vally IM, Vally U (2019) Maxillofacial Trauma: Peri Operative Challenges in the Management of Facial Bone Fractures. Int J Anesth Pain Med. Vol.5 No.1:3

\section{Sports related injuries, falls and industrial accidents are other remaining causes}

The skeletal region of isolated facial bone fractures: red, frontal bone $(0.4 \%)$; yellow, orbital bone $(9.2 \%)$; green, nasal bone (45.9\%); blue, maxilla (1.6\%); purple, zygoma (6.9\%); orange, mandible (35.9\%) [6].

Purple area is made of dense bone (buttresses).

Energy distribution to the brain is protected by buttresses (Figure 1).

The mid face is made of the maxilla, zygoma and lower half of the naso-orbito-ethmoidal complex. Facial fractures occur where the

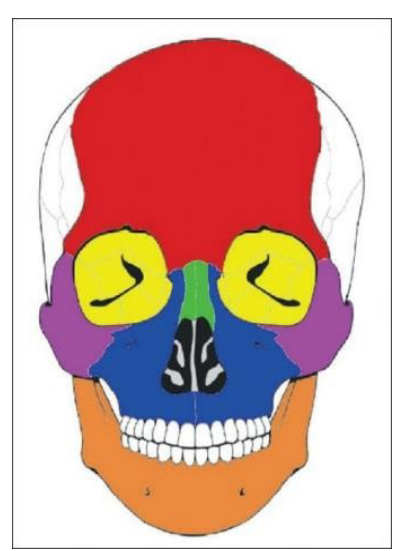

Figure 1 Skeletal Regions of the Face. 
bones are weakest and act as a crumple zone to protect the brain from injury [1].

Mogajane and Mabongo report the mandible (73\%) as the most frequent fractured bone followed by the zygoma [2] which is in keeping with international trends.

Traumatic high energy facial fractures are frequently associated with other significant injury. Traumatic brain injury occurs in maxillo-facial trauma. Joshi et al report an incidence of $67 \%$ in their study with concussion being the most common head injury [7]. Orbital, soft tissue and dental injuries are also common and there may be associated intracranial and cervical spine injury $[6,8]$.

Pan facial fractures are defects involving 3 or more facial units.

A simple Classification is to divide the face in equal thirds: Lower, middle and upper third respectively.

Le Fort 1; 2 and 3 classification involving the Maxilla (Figure 2).

\section{Case Report}

An adult female passenger was involved in a minibus taxi collision and suffered severe facial injuries. Minibus transport is a common, inexpensive and convenient mode of transport but have a poor safety track record. Overcrowding is common, seatbelts not available or enforced, speeding and disregard of road safety rules a common practice.

Initial assessment and investigation of the injuries in the trauma unit ruled out any cervical spine or traumatic brain injury and a Glasgow coma scale (GCS) of 15 was recorded.

In view of the severe facial injury and swelling and the likelihood of the airway being compromised an urgent tracheotomy was done under general anaesthesia.

Further management protocols included:

- Antibiotics prophylaxis for wound contamination and intraoral sepsis.

- Antacid therapy and antiemetic's to prevent stress ulcers, nausea and vomiting.

- Anti-epileptic medication and steroids to reduce cerebral contusion and prevent convulsions.

- Adequate analgesia and sedation to reduce the stress response.

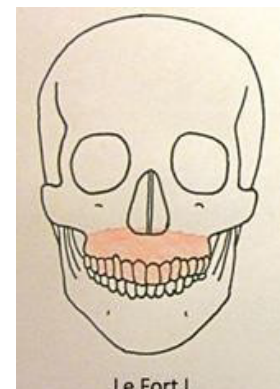

Le Fort I
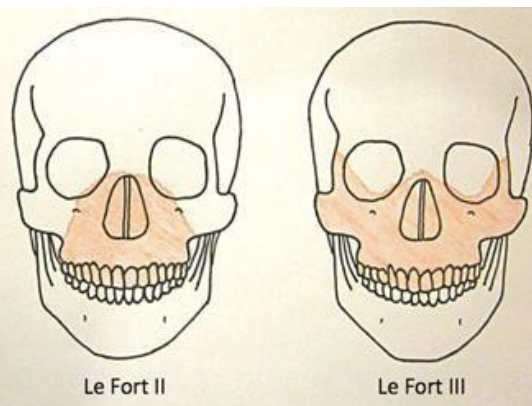

Figure 2 Le Fort 1; 2 and 3 classification involving the Maxilla.
- Stabilizing of vital signs with adequate replacement of fluid and volume loss.

No definitive surgical procedures were undertaken as surgery for facial bone fractures can safely be planned as an elective procedure once the swelling and oedema has reduced. Delays of up to a week makes for an easier operative field and better surgical outcome.

Emergency surgery is indicated only in cases of massive bleeding or if there is concurrent life- threatening injuries (Figure 3) [9].

Fractures involved included:

1. Left orbital - Lateral wall and orbital floor blowout injury with muscle entrapment.

2. Mandible - Comminuted fracture of the body symphysis.

3. L. Maxilla and Zygomatic arch displacement and fracture.

\section{Investigations}

- Blood $\mathrm{Hb}$ was $9.4 \mathrm{~g} / \mathrm{dl}$.

- Leucocyte count and CRP were high. Serum electrolytes were normal.

- Cardiac enzymes were elevated in keeping with cardiac contusion but soon reverted to normal.

Reference: (i) $\mathrm{S}-\mathrm{CKMB}=19$ (0-5). (ii) Troponin $\mathrm{T}=17$ (<15). (iii) S-Creatinase kinase $=847$ (26-140).

Respiratory support with synchronous intermittent mandatory ventilation (SIMV) was given. Gas exchange remained good and blood gas analysis normal.

A high-resolution CT scan further confirmed no cerebral bleeding or cervical spine injuries.

Vital signs remained stable with no signs of pulmonary contusion, viscus perforation or long bone fractures present.

Parental feeding was started and surgery scheduled electively a week later.

\section{Anaesthesia}

Gas induction was commenced with Nitrous Oxide/Oxygen $50 \%$.
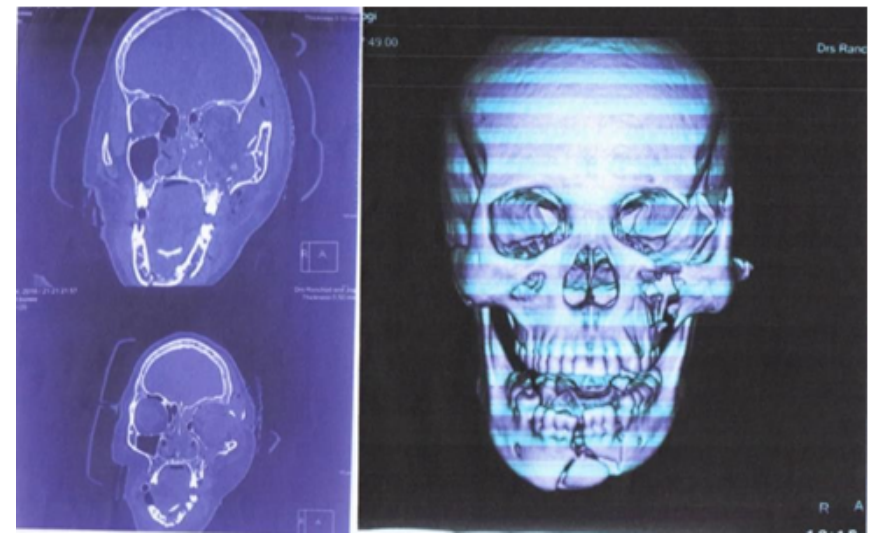

Figure 3 Before surgery and Pan facial Fracture. 
Rocuronium (9 $\mathrm{mg} / \mathrm{kg}$ ) used as muscle relaxation and given infusions of dexmedetomidine $(0.4 \mathrm{mg} / \mathrm{kg} / \mathrm{min})$ and remifentanil target-controlled infusions ( $\mathrm{TCl})(2-4 \mathrm{ng} / \mathrm{ml})$ using a Braun infusion system. Ketamine $(0.3 \mathrm{mg} / \mathrm{kg} / \mathrm{hr})$ a NMDA antagonist was added to prevent tolerance and hyperalgesia associated with remifentanil.

Dexmedetomidine and alpha2 adrenoreceptor agonist has analgesic properties and is useful in the control of blood pressure and the reduction of blood loss. Remifentanil an ultra- short acting opiate gives excellent analgesia and is readily titratable [10].

Surgery lasted 3 hours with open reduction and internal fixation of the fracture sights with miniplates. This method gives rapid osteosynthesis and stability and is technically easy to apply. Postoperatively the patient was sent to the intensive care unit (ICU) and kept on SIMV and given respiratory support. Enteral feeding was commenced with a gastro-enteral feeding tube in place. Dexmedetomidine together with ketamine at a sub anaesthetic $(0.5 \mathrm{mg} / \mathrm{kg})$ offer good sedation and analgesia and was continued as infusions in the ICU.

Opiate analgesia post operatively was avoided for its respiratory depressant effects and associated constipation, nausea and vomiting. A multimodal approach using anti-inflammatories and intravenously paracetamol was given for analgesia. Steroids which help in reducing inflammation and antibiotics were continued into the postoperative period.

The patient made remarkably good progress and was safely extubated and discharged a week later.

Facial bone fractures have a low complication rate $(<10 \%)$. The most common complaint is one of hypoesthesia and diplopia with the vast majority improving by 1 month while some requiring a longer time (Figure 4) [6].

-Plating of Left Orbital rim and infraorbital floor graft with plating.

- L. Maxillary plating and reduction.

- Mandible plating and reduction of comminuted fracture.

\section{Discussion}

Airway control and excluding cervical spine injury are priorities

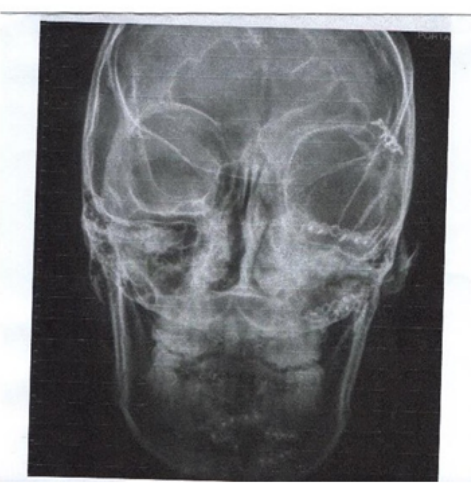

Figure 4 Post reconstruction. in the emergency management following maxillo-facial trauma.

Pan facial fractures pose unique anaesthetic challenges in securing a safe airway.

Complicating factors include a distorted facial appearance, a full stomach, the presence of blood, vomitus and secretions in the airway and bleeding that may be life threatening [1].

Acute airway obstruction can cause hypoxia leading to hypoxaemia, brain injury and death.

\section{Airway assessment and management}

Independent risk factors for difficult intubations include a limited jaw opening, a short thick neck circumference (>40 cms), Mallampati class 3-4, limited neck mobility and obesity (BMI $>35 \mathrm{~kg} / \mathrm{m} 2$ ) [1]. In managing intubations, a number of options are available. Listed below are useful methods. They are not exhaustive but developing competence in these essential [9].

1. Direct laryngoscopy with manual in -stabilization of the neck is easy when no difficulties are anticipated.

2. Airway Rescue devices: laryngeal mask airway (LMA) can be used in the cannot intubate cannot ventilate (CICV) situation until a definite airway is established.

3. Glide-scope video-laryngoscope intubation. This is a major advance in airway management.

4. Fibreoptic (FO) guided nasotracheal intubation in patients with very limited mouth opening.

5. Cricothyroidotomy and tracheostomy are choices for emergency airway control.

\section{Assessment of neurological injury}

GCS is a clinical assessment of patients with acute head trauma [11]. It is scored on

1. Eye movements (1-4)

2. Verbal response (1-5)

3. Pain response (1-6)

A score of 8 or less is suggestive of serious brain injury and cognitive dysfunction.

- Scores (9-12) moderate head injury.

- Scores (13-15) mild cognitive deficit.

Midfacial injuries and pan facial fractures are associated with serious head injuries and this need to be excluded. Initial screening of a GCS should be followed by later neural -imaging studies and a neurological examination to evaluate true cognitive deficits.

Joshi et al report that concussion is one of the most commonly associated cognitive dysfunction with traumatic facial injuries and the risk of head injury increased significantly with an increased in the number of facial fractures and a decreased GCS score [7]. 


\section{Conclusion}

Emergency trauma personnel must be aware of the likelihood of brain injury and cervical spine injury in their initial assessment of mid -facial traumatic injury and as a priority ensures a safe and secure airway.

Interpersonal violence is the most common cause of maxillofacial fractures in developed countries followed by road accidents and this is also the conclusion in a recent study comparing an urban and rural population in SA. Violence prevention programs in communities with high criminality require strong law enforcement and community peace initiatives. Positive parenting, a safe home and community environment are important. Unemployment, poverty, the breakdown of family values, drug addictions and glorifying a culture of violence all contribute to violent criminal behaviour. Crime statistics in SA vary across geographical areas with areas having a ratio of 233/100 000 murders/4 yrs. being equal to some of the most violent capitals of the world.

\section{References}

1 Morosan M, Parbhoo A, Curry N (2012) Anaesthesia and common oral and maxillo-facial emergencies. Continuing Education in Anaesthesia Critical Care \& Pain 12: 257-262.

2 Mogajane BM, Mabongo M (2018) Epidemiology of maxillofacial fractures at two maxillofacial units in South Africa. SADJ 73: 132-136.

3 Crime statistics in South Africa (2018-2019). Crime statistics report released in September 2019. Police Commissioner Bheki Cele.

4 (2018) World Health Organisation. Global status report on road safety.

5 (2018) Road Traffic Management Corporation. Internet on Line. Natal Mercury Report.
MVA and road deaths in SA are a national crisis. Peaks occur over holiday periods despite road safety awareness campaigns. Human error, speeding, drunk driving, unroadworthy vehicles, driver fatigue and poor judgement are contributory factors. The cost to the SA economy translates to $3.4 \%$ of gross domestic product (GDP) which is $1.4 \%$ higher than the international benchmark.

Road accidents have horrific long-term consequences for individuals, family and society causing permanent disability, loss of productivity and death. AA of SA report 14050 deaths in road accidents on South African roads in 2017.

A Multifactorial approach that incorporates individuals, communities and society at large with government intervention and law enforcement is required to curb interpersonal violence and road carnage. Emergency medical teams and trauma units available at short notice are being deployed in all major centres in South Africa.

6 Hwang K, You S (2010) Anallysis of Facial Bone Fractures: an 11 year study of 2094 patients. Indian J Plastic Surg 43: 42-48

7 Joshi UM, Ramdurg S, Saiker S, Patil S, Shah K (2018) Brain Injuries and Facial Fractures: A Prospective Study of Head Injury Associated with Maxillofacial Trauma. J Maxillofac Oral Surg 17: 531-537.

8 Steve C, Hogrefe C (2019) Diagnosis and Management of Facial Bone Fractures. Emerg Med Clin N Am 37: 137-151.

9 Gupta B, Singh S, Kumar K (2017) Anaesthetic Challenges and Management of Maxillofacial Trauma. Journal of Anaesthesia and Surgery. J Anesth Sur 17: 12-02.

10 (2018) South African Society of Anaesthesiology. Congress TCI workshop. Problem based discussions.

11 Whitfield PC, Thomas EO, Whyte M (2009) Head Injury: A Multidisciplinary Approach. Cambridge Medicine. 